\title{
Fluxos Ramificados Arco-disjuntos em Redes de Capacidade Restrita*
}

\author{
A. Karolinna Maia ${ }^{1}$, Jonas Costa ${ }^{1,2}$, Raul Lopes ${ }^{1,2}$ \\ ${ }^{1}$ Departamento de Computação - Universidade Federal do Ceará (UFC) \\ Grupo de Pesquisa ParGO \\ Fortaleza - CE - Brazil \\ karolmaia@lia.ufc.br, jonascosta@lia.ufc.br, raul.wayne@gmail.com
}

\begin{abstract}
The problem of determining if a given network has a feasible flow is largely studied and known to be polynomial-time solvable. In this work, we consider an specific type of flow, called branching flow, which can be useful for finding branchings in digraphs. We focus on the problem of finding multiple arc-disjoint branching flows on a given network and we study its complexity on networks with different capacities on the arcs. A previous result shows that, under a common theoretical assumption (ETH), in networks with $n$ vertices where all the arcs have capacity equal to $n-f(n)$, for a bounded function $f$, this problem is hard. We extended this result showing that, under the same assumption, the problem is also hard when the capacities are equal to $f(n)$.
\end{abstract}

Resumo. Determinar se uma rede possui um fluxo viável é um problema amplamente estudado e que pode ser resolvido em tempo polinomial. Investigamos um tipo específico de fluxo, o fluxo ramificado, que pode ser aplicado na busca por ramificações em digrafos. Consideramos o problema de encontrar múltiplos fluxos ramificados arco-disjuntos em uma rede e estudamos a complexidade deste problema com diferentes capacidades nos arcos. Um resultado anterior prova que, sob uma suposição teórica conhecida (ETH), em redes com $n$ vértices tais que todos os arcos possuem capacidade $n-f(n)$, para uma função limitada $f$, o problema é difícil. Estendemos este resultado mostrando que, sob a mesma hipótese, o problema também é difícil quando as capacidades são iguais a $f(n)$.

\section{Introdução}

Neste trabalho, seguimos a terminologia básica contida em [Bang-Jensen and Gutin 2008]. Uma rede $\mathcal{N}=(V, A, u)$ é definida por um digrafo $D=(V, A)$ e uma função de capacidade $u: A \rightarrow \mathbb{Z}_{+}$. Usamos a notação $u_{v w}$ para nos referir a $u(v w)$, para todo arco $v w \in A$. Podemos escrever $\mathcal{N}=(V, A, u \equiv l)$ quando a capacidade de todos os arcos da rede é $l$.

Um fluxo em uma rede $\mathcal{N}$ é uma função $x: A \rightarrow \mathbb{Z}_{+}$, onde $x_{v w}$ denota o valor de $x$ no arco $v w$. O balanço de um fluxo $x$ é uma função que atribui a cada vértice $v \in V$ o valor

$$
b_{x}(v)=\sum_{v w \in A} x_{v w}-\sum_{z v \in A} x_{z v}
$$

\footnotetext{
${ }^{2}$ Financiado pela CAPES.

*Este trabalho foi parcialmente apoiado pelo CNPq - projeto Universal 425297/2016-0 e FUNCAP PRONEM PNE-0112-00061.01.00/16.
} 
Um fluxo $x$ em $\mathcal{N}$ é dito viável se $x_{v w} \leq u_{v w}$, para todo arco $v w \in A$. Frenquentemente, no estudo do problema de fluxo é considerado a sua conservação, isto é, $b_{x}(v)=0$ para todo vértice $v \in V$.

Fluxos em redes são amplamente estudados, pois além de modelarem problemas práticos de grande importância, também despertam grande interesse teórico sendo várias vezes uma ferramenta útil para a resolução de problemas em grafos e digrafos. Uma vasta coleção de resultados de fluxo é apresentada em [Ahuja 1993].

As variações mais conhecidas de problemas de fluxo podem ser resolvidas em tempo polinomial. No entanto, existem problemas de otimização que lidam com caminhos e ciclos em digrafos e se assemelham tipicamente a fluxos, mas que não podem ser modelados em sua forma tradicional. Um exemplo clássico é o problema de encontrar $k$ caminhos arco-disjuntos em um digrafo $D$. Neste, recebemos como entrada $2 k$ vértices $s_{1}, s_{2}, \ldots, s_{k}, t_{1}, t_{2}, \ldots, t_{k} \in V(D)$ e queremos decidir se existem $k$ caminhos arco-disjuntos $P_{1}, P_{2}, \ldots, P_{k}$ em $D$ tal que $P_{i}$ é um $\left(s_{i}, t_{i}\right)$-caminho para $i \in\{1,2, \ldots, k\}$. Este problema foi mostrado ser $\mathcal{N} \mathcal{P}$-completo em [Fortune et al. 1980] para $k \geq 2$. Com o objetivo de obter uma estrutura mais geral e abranger alguns desses casos, [Bang-Jensen and Bessy 2014] começaram a investigar a existência múltiplos fluxos viáveis arco-disjuntos em uma rede pré-determinada. Dois fluxos $x$ e $y$ em $\mathcal{N}$ são ditos arco-disjuntos se $x_{v w} \cdot y_{v w}=0$ para todo arco $v w \in A$.

Uma ramificação em um digrafo $D$ é uma árvore geradora de $D$ na qual todos os vértices, com exceção de um chamado de raiz, possuem grau de entrada um. Uma $s$-ramificação é uma ramificação cuja a raiz é o vértice $s$. O estudo de ramificações em digrafos também possui motivações práticas. Por exemplo, em um contexto em que se deseje enviar informações de uma fonte para todos os demais vértices do digrafo, os arcos de uma ramificação podem ser utilizados para fazer o roteamento das mensagens. Porém, essas estruturas podem não ser muito seguras. Por esse motivo se torna interessante o estudo de medidas de qualidade de uma ramificação. Alguns exemplos de parâmetros considerados podem ser vistos em [Bang-Jensen and Yeo 2015, Bang-Jensen et al. 2016].

Um fluxo s-ramificado em uma rede $\mathcal{N}=(V, A, u)$ é um fluxo $x$ no qual $b_{x}(s)=$ $n-1$ e $b_{x}(v)=-1$ para todo $v \in V \backslash\{s\}$, onde $n$ é o número de vértices de $\mathcal{N}$. Neste tipo de fluxo cada vértice da rede recebe pelo menos uma unidade de fluxo. Observe que o subgrafo de $\mathcal{N}$ definido por $V$ e pelos arcos em que o valor de $x \neq 0$ não necessariamente é uma $s$-ramificação.

Em [Bang-Jensen and Bessy 2014], os autores introduzem o problema de decidir se existem 2 fluxos $s$-ramificados arco-disjuntos em uma rede $\mathcal{N}$, e [Bang-Jensen et al. 2016] se aprofundaram nessa questão, considerando diferentes capacidades para $\mathcal{N}$. No presente trabalho, resolvemos um importante caso deixado em aberto relativo ao mesmo problema, além de termos obtido uma prova alternativa do caso complementar apresentada por [Bang-Jensen et al. 2016]. Devido a restrições de espaço, todas as provas serão omitidas.

\section{Fluxos ramificados em redes com capacidades específicas}

Através de uma prova algorítmica dada por [Lovász 1976] para um teorema originalmente descrito por [Edmonds 1973], é possível obter um algoritmo polinomial para 
decidir se uma rede $\mathcal{N}=(V, A, u \equiv n-1)$ admite $k$ fluxos $s$-ramificados $x^{1}, x^{2}, \ldots, x^{k}$ arco-disjuntos. Reduzir a capacidade dos arcos por um fator constante não é suficiente para alterar a dificuldade do problema quando consideramos apenas dois fluxos:

Teorema 1. [Bang-Jensen et al. 2016] Para todo inteiro positivo $k$ fixo, existe um algoritmo que recebe como entrada uma rede $\mathcal{N}=(V, A, u \equiv n-k)$ e um vértice especial $s$ e decide em tempo polinomial se $\mathcal{N}$ admite dois fluxos s-ramificados arco-disjuntos.

Um resultado de [Bang-Jensen and Bessy 2014] mostra que é polinomial decidir se uma rede com capacidade unitárias $(u \equiv 1)$ possui dois fluxos arco-disjuntos com o mesmo balanço. Note que, em particular, o resultado vale para fluxos ramificados. Por outro lado, o seguinte teorema foi provado:

Teorema 2. [Bang-Jensen and Bessy 2014] É $\mathcal{N} \mathcal{P}$-completo decidir se uma rede $\mathcal{N}=$ $(V, A, u)$, com $u_{v w} \in\{1,2\} \forall v w \in A$, possui dois fluxos s-ramificados arco-disjuntos.

O Teorema 2 é utilizado para provar que se aumentarmos a capacidade em no máximo uma constante o problema continua $\mathcal{N} \mathcal{P}$-completo.

Teorema 3. [Bang-Jensen et al. 2016] Para todo inteiro $k \geq 2$ fixo, é $\mathcal{N} \mathcal{P}$-completo decidir se uma rede $\mathcal{N}=(V, A, u \equiv k)$ possui dois fluxos s-ramificados arco-disjuntos.

Pelos resultados anteriores, observamos que, aparentemente, ao aumentarmos a capacidade das arestas de $\mathcal{N}$, o problema de encontrar fluxos arco-disjuntos pode apenas se tornar mais fácil. Dessa forma, conjecturamos que deve existir algum valor $\ell \in\{2, \ldots, n-1\}$ onde é difícil decidir se uma rede com arcos de capacidade $u \leq \ell$ admite dois fluxos $s$-ramificados e arco-disjuntos, porém o problema é fácil para capacidades maiores (Teorema 1).

Estendemos resultados obtidos em [Bang-Jensen et al. 2016] para mostrar que, como consequência da Hipótese de Tempo Exponencial (ETH) [Impagliazzo et al. 2001], o problema considerado é difícil para quase todos os valores possíveis de $\ell$. A ETH é uma conjectura que afirma não existir algoritmo para resolver o problema 3-SAT que seja sub-exponencial no número de variáveis.

Mostramos que a existência de um algoritmo polinomial no número de vértices para o problema, quando consideramos as capacidades por uma função limitada de $n$, implicaria na existência de um algoritmos sub-exponencial para 3-SAT.

Teorema 4. Assumindo válida a ETH, seja $\epsilon>0$ arbitrário e $f(n)$ uma função inteira tal que $\log (n)^{1+\epsilon} \leq f(n) \leq n / 2$ para todo $n>0$. Além disso, suponha que existe uma constante $C^{*}$ no qual para todo $c \geq C^{*}$ existe um $n$ tal que $f(n)=c$. Dadas essas condições, seja $\mathcal{N}=(V, A, u \equiv f(n))$ uma rede com $n$ vértices e um vértice especial $s \in V$. Não existe algoritmo polinomial (em n) para decidir se $\mathcal{N}$ possui dois fluxos s-ramificados arco-disjuntos.

O Teorema 4, principal contribuição deste trabalho, considera o caso de redes com capacidades $f(n)$. A demonstração segue uma adaptação da demonstração para o caso de redes com capacidades $n-f(n)$ contida em [Bang-Jensen et al. 2016]. Ressaltamos que, com algumas alterações, a prova oferecida para o Teorema 4 se mostra 
como uma prova alternativa para o resultado já conhecido para redes com capacidades $n-f(n)$. A Figura 1 ilustra para quais capacidades a complexidade do problema considerado é conhecida. Segmentos contínuos marcam resultados previamente conhecidos ([Bang-Jensen et al. 2016, Bang-Jensen and Bessy 2014]) e o segmento curvo marca nossa contribuição.

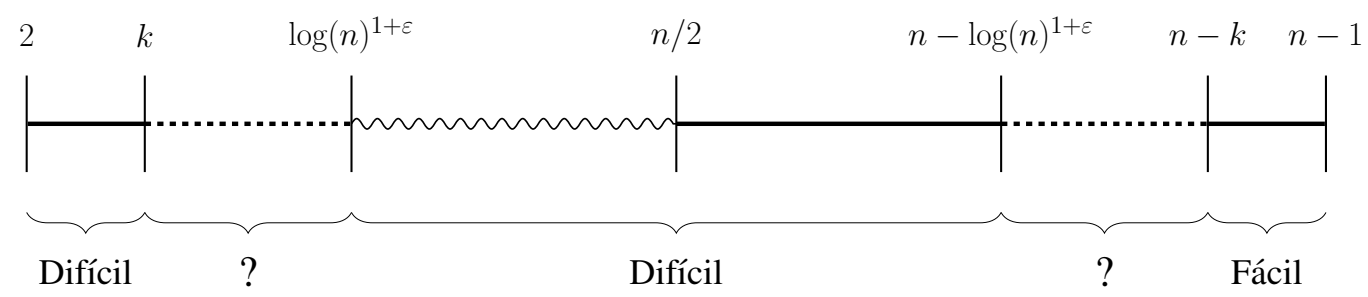

Figura 1. Intervalos de capacidades e complexidades.

\section{Referências}

Ahuja, R. (1993). Network flows : theory, algorithms, and applications. Prentice Hall, Englewood Cliffs, N.J.

Bang-Jensen, J. and Bessy, S. (2014). (Arc-) disjoint flows in networks. Theoretical Computer Science, 526:28-40.

Bang-Jensen, J. and Gutin, G. Z. (2008). Digraphs: theory, algorithms and applications. Springer Science \& Business Media.

Bang-Jensen, J., Havet, F., and Yeo, A. (2016). The complexity of finding arc-disjoint branching flows. Discrete Applied Mathematics, 209:16-26.

Bang-Jensen, J. and Yeo, A. (2015). Balanced branchings in digraphs. Theoretical Computer Science, 595:107 - 119.

Edmonds, J. (1973). Edge-disjoint branchings. Combinatorial Algorithms, pages 91-96.

Fortune, S., Hopcroft, J., and Wyllie, J. (1980). The directed subgraph homeomorphism problem. Theoretical Computer Science, 10(2):111 - 121.

Impagliazzo, R., Paturi, R., and Zane, F. (2001). Which problems have strongly exponential complexity? Journal of Computer and System Sciences, 63(4):512 - 530.

Lovász, L. (1976). On two minimax theorems in graph. Journal of Combinatorial Theory, Series B, 21(2):96 - 103. 